\title{
A PINCHING THEOREM FOR HOMOTOPY SPHERES
}

\author{
KARSTEN GROVE AND PETER PETERSEN V
}

The (length) excess of a triangle measures how much the triangle inequality fails to be an equality. This notion was first studied seriously in [AG]. We say that a (bounded) metric space $X=(X, d)$ has excess $\leq \varepsilon$ if there are points $p, q \in X$ such that $d(p, x)+d(x, q) \leq d(p, q)+\varepsilon$ for all $x \in X$. The excess, $e(X)$, of $X$ is the infimum of all $\varepsilon \geq 0$ where $X$ has excess $\leq \varepsilon$. Notice that the standard spheres have excess $=0$. In general, a closed Riemannian manifold $M$ has excess $=0$ if and only if there is a point $p \in M$ whose cutlocus $C(p)$ is a single point $q \in M$. Such a manifold is clearly a twisted sphere, i.e., it is the union of two discs glued together along their common boundary. Conversely according to a result of Weinstein [B; Appendix C.4], any twisted sphere carries a Riemannian metric for which there is a point whose cut-locus is another point.

With this in mind, we are interested in closed Riemannian manifolds with small excess. However, without any further geometric restrictions, small excess has no topological significance (see Problem 8, though). In fact, any closed $n$-manifold $M$ can be given a Riemannian metric with $\operatorname{diam} M=\pi$ and arbitrarily small excess. Simply make sure that all topological complexities of $M$ (i.e., the complement of a topological $n$-disc) are contained in a sufficiently small metric ball and what remains is metrically close to the complement of a small ball in the unit sphere $S^{n}(1)$. Our main result is

Theorem A. Given $k \in \mathbf{R}, D, v>0$, and an integer $n \geq 2$, there is an $\varepsilon=$ $\varepsilon(n, k, D, v)$ such that any closed Riemannian $n$-manifold $M$ with sectional curvature $K_{M} \geq k$, $\operatorname{diam} M \leq D$, Vol $M \geq v$, and $e(M) \leq \varepsilon$ is a homotopy sphere.

The following three examples of families of metrics on the 2-torus show that Theorem $\mathbf{A}$ is optimal.

Example I. Let $S^{n}(r)$ denote the round $n$-sphere in $\mathbf{R}^{n+1}$ with radius $r$. The connected sums $M_{r}^{2}=S^{2}(1) \# S^{1}(r) \times S^{1}(r), r \rightarrow 0$, provide a family of Riemannian manifolds with $K_{M_{r}^{2}} \leq 1$, diam $M_{r}^{2} \rightarrow \pi$, Vol $M_{r}^{2} \rightarrow 4 \pi$ and $e\left(M_{r}^{2}\right) \rightarrow 0$. This shows that the lower curvature bound is needed.

Received by the editors November 14, 1989.

1980 Mathematics Subject Classification (1985 Revision). Primary 53C20.

Supported in part by grants from the National Science Foundation.

(C) 1990 American Mathematical Society 
Example II. The family $N_{r}^{2}=S^{1}(r) \times S^{1}\left(\frac{1}{r}\right), r \rightarrow 0$, of flat tori satisfy Vol $N_{r}^{2}=$ $4 \pi^{2}$ and $e\left(N_{r}^{2}\right) \rightarrow 0$. Hence the diameter assumption is necessary.

Example III. To see that one needs the volume assumption, consider the family $V_{r}^{2}=S^{1}(r) \times S^{1}(1), r \rightarrow 0$, of flat tori. Clearly diam $V_{r}^{2} \rightarrow 2 \pi$ and $e\left(V_{r}^{2}\right) \rightarrow 0$.

Our original motivation for considering the excess of a metric space was that a closed Riemannian manifold $M^{n}$ with Ricci curvature $\mathrm{Ric}_{M} \geq n-1$ and $\operatorname{diam} M$ close to $\pi$ has small excess (Lemma 1). As an immediate consequence of Theorem A we therefore have

Corollary B. Given $k \in \mathbf{R}, v>0$ and an integer $n \geq 2$. There is a $d=$ $d(n, k, v)<\pi$ such that any complete Riemannian n-manifold $M$ with $K_{M} \geq$ $k, \operatorname{Vol} M \geq v, \operatorname{Ric} M \geq n-1$ and $\operatorname{diam} M \geq d$ is homeomorphic to the $n$ sphere.

In addition to Theorem A, we must here use Hamilton's classification of 3manifolds with positive Ricci curvature $[\mathrm{H}]$ and the generalized Poincare conjecture [F, Sm]. This corollary generalizes the main results in [Sh, I, W, and E]. In each of these papers, the geometric assumptions are strong enough to guarantee that either the injectivity radius or at least the criticallity radius (called contractibility radius by most authors following [Sh]) of $M$ is bounded from below. This however, is not the case with our assumptions:

Example IV (thin spheres). Given $v \in\left(0, \frac{1}{2} \operatorname{Vol} S^{n}\right), d \in\left(\frac{\pi}{2}, \pi\right)$, it is not hard to construct Riemannian metrics $g_{v, d}$ on $S^{n}$ with sectional curvature $\geq 1$, volume $=v$ and diameter $=d$. If we fix $v$ and let $d \rightarrow \pi$, these metrics will converge to a singular metric on $S^{n}$, which has diameter $=\pi$, singularities at north and south poles and sectional curvature $=1$ outside the singularities. Suppose that $v \ll \operatorname{Vol} S^{n}$. Then the metrics $g_{v, d}$ will develop very sharp conical singularities at $N$ and $P$ as $d \rightarrow \pi$. Thus there is no lower bound for injectivity or contractibility radius for the family $\left\{S^{n}, g_{v, d}: v\right.$ fixed small, $\left.d \in\left(\frac{\pi}{2}, \pi\right)\right\}$.

We point out that recent examples of Anderson [A] and Otsu [O] indicate that also our Corollary B may be optimal. In fact there are manifolds $M^{n} \neq S^{n}$ with $\operatorname{Ric} M \geq n-1, \operatorname{Vol} M \geq v$ and $\operatorname{diam} M$ arbitrarily close to $\pi$.

An illuminating and, in spirit, simple existence proof of $\varepsilon$ in Theorem A can be given based on the machinery developed in [P, GPW and GP2]. If no such $\varepsilon$ exists we find a sequence of manifolds $M_{i}$ with $K_{M_{i}} \geq k, \operatorname{diam} M_{i} \leq D$ and Vol $M_{i} \geq v$ such that the Gromov-Hausdorff limit space $X$ of $\left\{M_{i}\right\}$ has excess, $e(X)=0$. Since also $X$ has Toponogov curvature $\geq k$ [GP2], $X$ must be a suspension $X=\Sigma E$ (see also [GP3]). From [GPW] we know that $X$ is a generalized manifold, hence simply connected and, by Poincare duality, a homology sphere. However, for $i$ large enough, $M_{i}$ is homotopy equivalent to $X$ [P], i.e., $M_{i}$ is a homotopy sphere. 
The purpose of this paper is to give an elementary constructive proof of Theorem A and Corollary B. This proof has the advantage of providing (in principle) an explicit estimate for $\varepsilon$.

\section{Closed MANifolds With SMALl EXCESS}

We begin by proving that $n$-manifolds with Ricci curvature $\geq n-1$ and diameter close to $\pi$ have small excess. We then go on to prove two lemmas relating excess to lower sectional curvature bounds and bounds on the diameter. These are then used together with [GP1, 1.3] to prove Theorem A. All proofs are constructive and can in principle be used to give explicit estimates. To fix notation we let $B(p, r) \subset M$ be the closed metric $r$-ball in $M$ centered at $p$, and $V(n, r)$ the volume of an $r$-ball in $S^{n}(1)$.

Lemma 1 (cf. also [E]). Let $M$ be a complete Riemannian n-manifold with $\operatorname{Ric}(M) \geq n-1$ and $\operatorname{diam}(M)=D$. If $p, q \in M$ with $d(p, q)=D$ and $\alpha+\beta=D$, then $M=B(p, \alpha+\varepsilon) \cup B(q, \beta+\varepsilon)$ whenever $V(n, \varepsilon) \geq V(n, D)-$ $2 V\left(n, \frac{D}{2}\right)$. In particular $e(M) \leq 2 \varepsilon$.

Proof. Suppose on the contrary that there is $x \in M$ with $d(x, p) \geq \alpha+\varepsilon$ and $d(x, q) \geq \beta+\varepsilon$. Then the balls $B(p, \alpha), B(q, \beta)$ and $B(x, \varepsilon)$ are pairwise disjoint. Hence

$$
\begin{aligned}
\operatorname{Vol}(M) & \geq \operatorname{Vol} B(x, \varepsilon)+\operatorname{Vol} B(p, \alpha)+\operatorname{Vol} B(q, \beta) \\
& \geq \frac{\operatorname{Vol} M}{V(n, D)}(V(n, \varepsilon)+V(n, \alpha)+V(n, \beta) \\
& \geq \frac{\operatorname{Vol} M}{V(n, D)}\left(V(n, \varepsilon)+2 V\left(n, \frac{D}{2}\right)\right) .
\end{aligned}
$$

Here the second inequality is the Bishop-Gromov volume comparison theorem (cf. e.g., [Gr]) and the third follows from the fact that $\alpha+\beta=D$. Consequently $V(n, \varepsilon) \leq V(n, D)-2 V\left(n, \frac{D}{2}\right)$, which is a contradiction.

This lemma, together with Theorem A, proves Corollary B. In the next two lemmas we fix a closed Riemannian manifold $M$ with $K_{M} \geq k, 0<d \leq$ $\operatorname{diam} M \leq D$, and $p, q \in M$ realizing the excess of $M$; i.e., $e(M)$ is the maximum of the excess function $e_{q, p}(x)=d(p, x)+d(x, q)-d(p, q)$. In this case we have

$$
\operatorname{diam} M \leq d(p, q)+e(M)
$$

by the triangle inequality.

Lemma 3. For every $\delta>0$ there is an $\varepsilon=\varepsilon(\delta, k, d)$ such that any $x \in$ $M-(B(p, \delta) \cup B(q, \delta))$ is a regular point for $p$ as well as for $q$ as long as $e(M) \leq \varepsilon$.

Proof. It suffices to prove that the angle between any minimal geodesic from $x$ to $p$ and from $x$ to $q$ is $>\frac{\pi}{2}$ (cf. also [GS]). Choose a comparison triangle $(\bar{p}, \bar{x}, \bar{q})$ in the simply connected two-dimensional space form $H_{k}^{2}$ with 


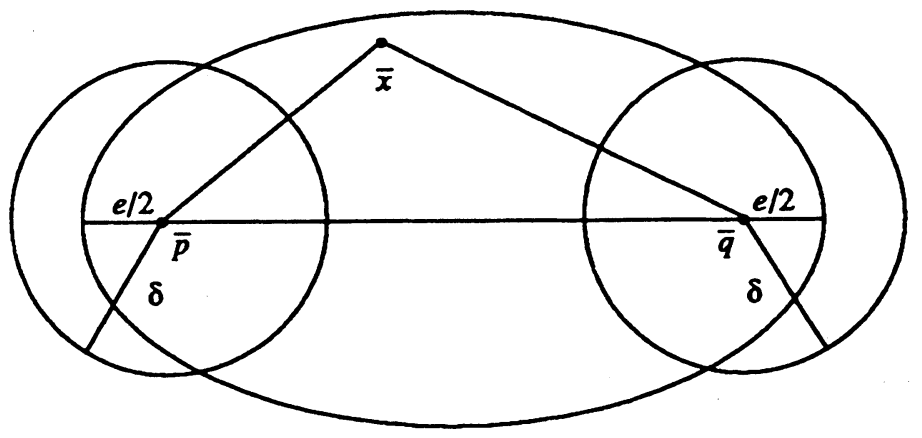

FIGURE 4

curvature $k$. By the Toponogov triangle comparison theorem, it is enough to show that the angle at $\bar{x}$ is $>\frac{\pi}{2}$. For each $d(\bar{p}, \bar{q}) \geq d-\delta$, there is an $\varepsilon=\varepsilon(\delta, k, d(\bar{p}, \bar{q})) \leq \delta$ such that for any $\bar{x}$ outside the $\delta$-balls at $\bar{p}$ and $\bar{q}$ inside the ellipse $\left\{x \in H_{k}^{2} \mid d(\bar{p}, \bar{x})+d(\bar{x}, \bar{q})=d(\bar{p}, \bar{q})+\varepsilon\right\}$ the angle $(\bar{p}, \bar{x}, \bar{q})$ is $>\frac{\pi}{2}$ (see Figure 4$)$. The worst estimate for $\varepsilon$ is clearly when $d(\bar{p}, \bar{q})=d-\delta$.

Lemma 5. For every $\delta \in\left(0, \frac{d}{10}\right)$, there is an $\varepsilon=\varepsilon(\delta, k, d, D) \in(0, \delta)$ and a $\Delta=\Delta(\delta, k, d, D)$ with $\lim _{\delta \rightarrow 0} \Delta=0$, such that if $e(M) \leq \varepsilon$ then

(i) $M-(B(p, \delta) \cup B(q, \delta))$ is connected, or

(ii) There is a geodesic biangle $B=\left(c_{p}, c_{q}\right)$ with $L(B)=L\left(c_{p}\right)+L\left(c_{q}\right) \in$ $[d, 3 D]$ such that any point in $M$ is within $\Delta$ of $B$.

Proof. From Lemma 3 it follows that there is a unit vector field on $M$ $(B(p, \delta) \cup B(q, \delta))$ such that for any integral curve $x(t), d(p, x(t))$ is strictly increasing and $d(q, x(t))$ is strictly decreasing. Thus if (i) does not hold, we can find $x_{1}, x_{2} \in M$ such that any curve from $x_{1}$ to $x_{2}$ must pass through $B(p, \delta) \cup B(q, \delta)$, and $d\left(x_{i},\{p, q\}\right) \geq \frac{1}{2} d(p, q) \geq \frac{1}{2}(d-e(M)), i=1,2$. For the last inequality we have used (2).

Let $c_{p}$ be a shortest curve from $x_{1}$ to $x_{2}$ passing through $B(p, \delta)$ and similarly for $c_{q}$, both parameterized by arclength. Since any curve from $x_{1}$ to $x_{2}$ must intersect either $B(p, \delta)$ or $B(q, \delta)$, both $c_{p}$ and $c_{q}$ are geodesics. Clearly $2 d-2 \varepsilon-4 \delta \leq L\left(c_{p}\right)+L\left(c_{q}\right) \leq 2 D+2 \varepsilon$. Now let $x \in M$. Then at least one minimal geodesic from $x_{1}$ to $x$ or from $x_{2}$ to $x$ must go through $B(p, \delta) \cup B(q, \delta)$. For the sake of argument, assume that $c:[0, l] \rightarrow M$ is a normal minimal geodesic from $x_{1}$ through $B(p, \delta)$ to $x$. Choose $t_{1}, t_{2} \geq$ $\frac{1}{2}(d-\varepsilon)-\delta \geq \frac{1}{3} d$ so that $c\left(t_{1}\right), c_{p}\left(t_{2}\right) \in B(p, \delta)$ and in particular $d\left(c\left(t_{1}\right), c_{p}\left(t_{2}\right)\right)$ $\leq 2 \delta$ (see Figure 6).

Since $L\left(c_{1}\right), L\left(c_{p}\right) \leq 2 D$ the Toponogov comparison theorem implies that $d\left(x, c_{p}\right) \leq d\left(c(l), c_{p}(l)\right) \leq \Delta$. Here $\Delta=\Delta(\delta, k, d, D)$ is the base of an 


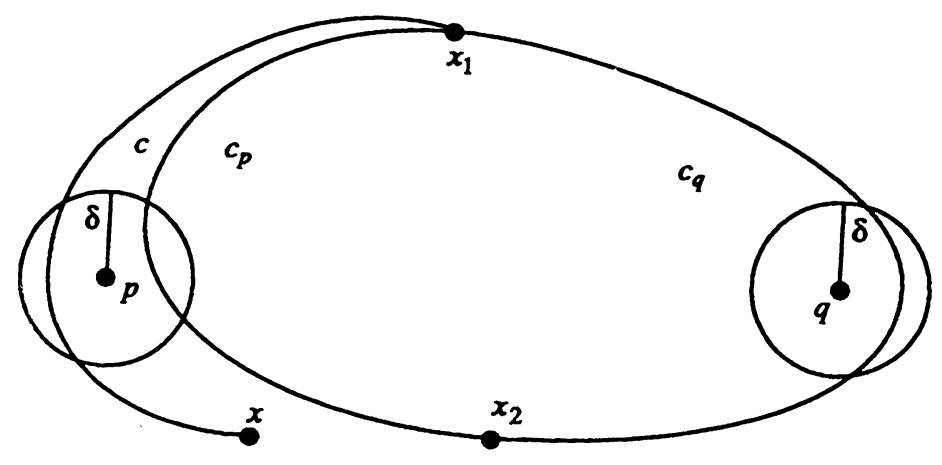

FIGURE 6

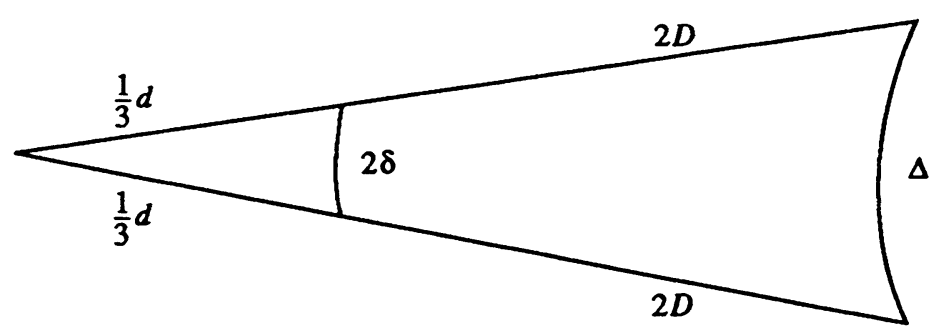

FIGURE 7

isosceles triangle in $H_{k}^{2}$ with sides $2 D$ which is the extension of an isosceles triangle with sides $\frac{1}{d}$ and base $2 \delta$ (see Figure 7).

Consider a closed Riemannian $n$-manifold $M$ with $K_{M} \geq k$, $\operatorname{diam} M \leq D$ and $\operatorname{Vol} M \geq v$. Then also $\operatorname{diam} M \geq d$, where the $d$-ball in $H_{k}^{n}$ has volume $v$.

Proof of Theorem A. The main lemma in [GP1] allows us to choose $\delta>0$ so that any $\delta$-ball in $M$ is contractible inside the concentric $\frac{d}{2}$-ball. By choosing $\varepsilon$ as in Lemma 3 we conclude that $M-B(q, \delta)$ contracts to $B(p, \delta)$ which in turn contracts to a point inside $B\left(p, \frac{d}{2}\right) \subset M-B(q, \delta)$. Similarly we see that $M-B(p, \delta)$ is contractible. Fix $\Delta_{0}$ so that the volume of a cylinder in $H_{k}^{n}$ with height $3 D$ and radius $\Delta_{0}$ plus the volume of two $\Delta_{0}$-balls in $H_{k}^{n}$ is $<v$. Choose $\delta$ so that in addition $0<\delta<\frac{d}{10}$ and $\Delta(\delta, k, d, D) \leq \Delta_{0}$. By possibly choosing $\varepsilon$ smaller according to both Lemmas 3 and 5 , we see that $M-(B(p, \delta) \cup B(q, \delta))$ is connected. Because otherwise $M$ would be contained in a $\Delta_{0}$ tube around a curve of length $\leq 3 D$, a simple volume comparison would then imply that $\operatorname{Vol} M<v$ by choice of $\Delta_{0}$. Thus $M$ is covered by two open contractible sets with connected intersection. $M$ is 
therefore simply connected by Van Kampen's theorem. Also all cup products between elements of degree $\geq 1$ vanish. Poincare duality then shows that $M$ is a homology sphere. Using theorems of Hurewicz and Whitehead, it then follows that $M$ has the homotopy type of $S^{n}$.

In view of [G] and Lemmas 3 and 5 above, it is apparent that there are topological restrictions on manifolds $M$ with $K_{M} \geq k, d_{M} \leq D$ and small excess.

Problem 8. Given $n \geq 2, k \in \mathbf{R}$ and $D>0$, describe the closed Riemannian $n$-manifolds $M$ with $K_{M} \geq k, d_{M} \leq D$ and arbitrarily small excess.

According to [Y], any orbit space $M / G$ of a compact manifold $M$ by a compact Lie group $G$ is the Gromov-Hausdorff limit of a sequence $\left\{M_{i}\right\}$ of Riemannian structures on $M$ with curvature bounded from below. In particular, any manifold $M$ of cohomogeneity one satisfies the conditions of Problem 8 above.

Remark 9. Without any bound on the diameter there are still constraints on manifolds with small excess and a lower curvature bound. Using arguments as in Lemma 3, one can show that there is a universal constant $\hat{e} \geq \frac{1}{2}$ such that any Riemannian manifold with $K_{M} \geq-1$ and $e(M) \leq \hat{e}$ has no critical points for points $p, q$ realizing the excess in the region $M-(B(p, 10) \cup B(q, 10))$. Such manifolds must therefore have a bound on the Betti numbers depending only on the dimension (cf. also [G, S]).

\section{REFERENCES}

[AG] U. Abresch and D. Gromoll, On complete manifolds with non-negative Ricci curvature, J. Amer. Math. Soc. 3 (1990), 355-374.

[A] M. Anderson, Metrics of positive Ricci curvature with large diameter, preprint, SUNY, Stony Brook.

[B] A. L. Besse, Manifolds all of whose geodesics are closed, Ergeb. Math. Grenzgeb., vol. 93, Springer-Verlag, 1978.

[E] J.-H. Eschenburg, Diameter, volume and topology for positive Ricci curvature, preprint.

[F] M. Freedman, The topology of four-dimensional manifolds, J. Differential Geom. 17 (1982), 357-453.

[G] M. Gromov, Curvature, diameter and Betti numbers, Comment. Math. Helv. 56 (1981), 53-78.

[Gr] K. Grove, Metric differential geometry, Differential Geometry, Proc. Nordic Summer School, Lyngby 1985 (ed. V. L. Hansen), Lecture Notes in Math., vol. 1263, Springer-Verlag, 1987, pp. 171-227.

[GP1] K. Grove and P. Petersen V, Bounding homotopy types by geometry, Ann. of Math. (2) 128 (1988), 195-206.

[GP2] _ Manifolds near the boundary of existence, J. Differential Geom. (to appear).

[GP3] __ Excess and rigidity of inner metric spaces, in preparation.

[GPW] K. Grove, P. Petersen V, and J.-Y. Wu, Controlled topology in geometry, Bull. Amer. Math. Soc. (N.S.) 20 (1989), 181-183; Geometric finiteness theorems via controlled topology, Invent. Math. 99 (1990), 205-213. 
[GS] K. Grove and K. Shiohama, A generalized sphere theorem, Ann. of Math. (2) 106 (1977), 201-211.

[H] R. S. Hamilton, Three-manifolds with positive Ricci curvature, J. Differential Geom. 17 (1982), 255-306.

[I] Y. Itokawa, The topology of certain Riemannian manifolds with positive Ricci curvature, J. Differential Geom. 18 (1983), 151-155.

[O] Y. Otsu, On manifolds of positive Ricci curvature with large diameter, preprint, Kyushu University.

[P] P. Petersen V, A finiteness theorem for metric spaces, J. Differential Geom. 31 (1990), 387395.

[S] Z. Shen, On the upper estimate of the Betti numbers of long Riemannian manifolds, preprint.

[Sh] K. Shiohama, A sphere theorem for manifolds of positive Ricci curvature, Trans. Amer. Math. Soc. 275 (1983), 811-819.

[Sm] S. Smale, Generalized Poincaré's conjecture in dimensions greater than four, Ann. of Math. (2) 64 (1956), 399-405.

[W] J.-Y. Wu, A diameter pinching sphere theorem for positive Ricci curvature, Proc. Amer. Math. Soc. 107 (1989), 797-802.

[Y] T. Yamaguchi, Collapsing and pinching in lower curvature bound, Ann. of Math. (to appear). 20742

Department of Mathematics, University of Maryland, College Park, Maryland

Department of Mathematics, University of California, Los ANgeles, California 90024 